\section{Helsinn: 20 years in primary cancer supportive care}

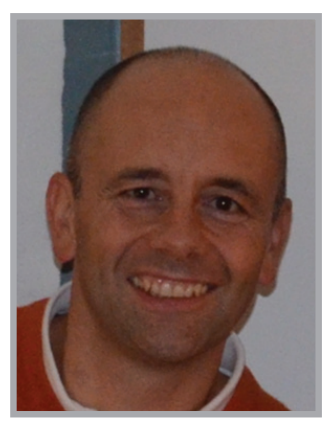

Sergio Cantoreggi* speaks to Henry Ireland, Commissioning Editor: Sergio Cantoreggi, $\mathrm{PhD}$, is the Chief Scientific Officer and Global Head of Research and Development of the Helsinn Group, a mid-sized pharmaceutical company headquartered in Lugano, Switzerland, and focused on providing cancer supportive care solutions to oncology patients worldwide. $\mathrm{Dr}$ Cantoreggi has overall responsibility for all R\&D activities of the Helsinn Group and has contributed to six major regulatory approvals of cancer supportive care agents in the USA, Europe and Japan. Dr Cantoreggi joined Helsinn Healthcare in 2000 as drug development scientist and was appointed Head of R\&D in 2005. In 2010, he was promoted to his current role. From 1994 to 2000 he worked as toxicologist and regulatory scientist for Du Pont, Sandoz and Novartis. Prior to joining industry, Dr Cantoreggi completed a postdoctoral fellowship and earned a Master of Science degree in chemistry and a Doctoral degree in natural sciences with a thesis on the mechanism of chemical carcinogenesis from the Swiss Federal Institute of Technology in Zürich, Switzerland.Sergio Cantoreggi discusses Helsinn's role in cancer supportive care, describing current treatment options for patients, the company's pipeline and Helsinn's work in supporting the field as a whole.

Accepted for publication: 21 September 2016; Published online: 3 November 2016

Q Can you tell us about your career \& how you became the Chief Scientific Officer of Helsinn?

After obtaining an MS degree in organic chemistry, I embraced the life sciences and did $\mathrm{my} \mathrm{PhD}$ and postdoctoral trainings investigating the mechanisms of carcinogenesis of several chemicals. This led me to an entry level position in industry as a toxicology study director, but already after 3 years I transitioned into a regulatory toxicologist/project management role. I then joined Helsinn where I was primarily responsible for preclinical studies but was also able to gain some experience in clinical development. After a few years I was offered the opportunity to take on overall responsibility for clinical development and regulatory affairs at the Helsinn Lugano site, at that time, the only Helsinn Research and Development (R\&D) hub. The role evolved significantly over the years and in 2010, I was promoted to Chief Scientific Officer and Global Head of R\&D. I am currently responsible for all R\&D and medical affairs' activities of the Helsinn Group overseeing a global team of approximately 120 highly qualified people spread over Switzerland, USA, Ireland and China.

\section{Q What are the main areas in cancer care that Helsinn is working on?}

Our main focus in the last years has been cancer supportive care, which is defined as the prevention and management of the adverse effects of cancer and its treatment. We

\section{KEYWORDS}

- cancer anorexia-cachexia • cancer supportive care

- chemotherapy-induced diarrhea

- chemotherapy-induced nausea and vomiting 
started this journey 20 years ago with the development and launch of the $5-\mathrm{HT}_{3}$ receptor antagonist palonosetron $\left(\mathrm{Aloxi}^{\circledR}\right)$ for the prevention of chemotherapy-induced nausea and vomiting (CINV). We then expanded into the treatment of chemotherapy-induced oral mucositis with Gelclair $^{\circledR}$ and recently received approval for netupitant and palonosetron (NEPA; Akynzeo ${ }^{\circledR}$ ), the only antiemetic product on the market combining a $5-\mathrm{HT}_{3}$ receptor antagonist (palonosetron) with an $\mathrm{NK}_{1}$ receptor antagonist (netupitant), simultaneously targeting two key pathways involved in CINV. Late stage $\mathrm{R} \& \mathrm{D}$ programs are focused on cancer cachexia and anorexia (anamorelin) and chemotherapy-induced diarrhea (CID; elsiglutide), both areas of high unmet medical need in cancer. We also have a clinical stage program in chemotherapy-induced neuropathic pain with HM01, a brain-penetrant ghrelin receptor agonist (GRA), the first compound invented by our research team. Besides these mainstream programs, we are exploring other potential indications for our pipeline products, in areas such as pruritus induced by targeted cancer therapies (e.g., EGFR inhibitors) and cancer fatigue through several academic collaborations.

People with cancer and cancer survivors are increasingly looking to manage better their symptoms and optimize their health status by accessing a growing market for medical devices, herbal extracts and medical foods. However, many of these products are not supported by clinical evidence and more than two-thirds of oncologists are unaware that their patients are using them. Last year we launched Helsinn Integrative Care, a business unit, which seeks to address a growing demand among people with cancer for evidence-based, nonpharmaceutical cancer supportive care products. Helsinn Integrative Care seeks to bring 'qualified' products to market which support the areas of highest patient needs, but backed with solid evidence.

Finally, we recently made a strategic decision to expand our reach beyond cancer supportive care and in August 2016, in-licensed pracinostat, an orally available histone deacetylase inhibitor that is being developed for advanced hematologic diseases such as acute myeloid leukemia and myelodysplastic syndromes.

\section{Q How important is industry partnership in providing cancer care?}

Despite the fantastic progress of the last decades in diagnosis and treatment, cancer remains a much dreaded disease with high mortality rates. The increased longevity of the world population, coupled with an increasing prevalence of predisposing factors such as obesity (particularly in developed countries) or smoking (particularly in developing countries), is expected to dramatically increase the number of cancer patients globally. All actors will be in high demand if we are to tackle this enormous global healthcare challenge across the continuum of the cancer experience. Industry will play a pivotal role in the development, manufacturing and distribution of innovative drugs, diagnostic and treatment technologies, and, in general, medical devices and supplies, to optimally address the needs of all cancer patients. Moreover, through the support of robust post registration and life cycle management programs, industry continuously contributes to generate new efficacy and safety data on marketed products, thus improving patients' care.

Q It is more than 10 years since the antiemetic drug, palonosetron, was made available for patients. How is this drug still impacting patient care today?

The introduction of the first-generation $5-\mathrm{HT}_{3}$ receptor antagonist ondansetron in the early 1990s has been a major breakthrough in cancer care affording for the first time a significant prevention of CINV, particularly in settings where highly emetogenic drugs such as cisplatin had to be used [1] with a much better side-effect profile in comparison with high-dose metoclopramide-dexamethasone [2]. Palonosetron is a new-generation $5-\mathrm{HT}_{3}$ receptor antagonist with a unique mode of action and a much longer half-life [3]. Palonosetron has demonstrated clinical superiority over first-generation drugs of the same class with a comparable safety profile [4-6]. These features have enabled palonosetron to become the preferred choice of many oncology healthcare practitioners worldwide. The use of palonosetron is also recommended by all treatment guidelines issued by the major international professional associations such as the American Society of Clinical Oncology, Multinational Association of Supportive Care in Cancer (MASCC) and the European Society for Medical Oncology [7-9]. The prevention of CINV has evolved over the last decade and in many settings; $5-\mathrm{HT}_{3}$ receptor antagonists are used in conjunction with corticosteroids, $\mathrm{NK}_{1}$ receptor antagonists and sometimes other drugs. 
Q Can you tell us more about your fixed-dose combination, netupitant-palonosetron?

CINV has a multifactorial origin, involving several neurotransmitters and receptors. International antiemetic guidelines recommend the use of combination regimens over several days to prevent CINV in many clinical settings. Although data support that this will improve CINV control for patients [10], adherence to guidelines is still suboptimal. NEPA is an oral-fixed single dose combination of a new $\mathrm{NK}_{1}$ receptor antagonist (netupitant) with a long half-life, pharmacologically distinct and clinically superior $5-\mathrm{HT}_{3}$ receptor antagonist (palonosetron), developed to enhance the convenience of administering guideline-based antiemetic prophylaxis by targeting the two major molecular pathways involved in the neuropharmacology of CINV. The efficacy of NEPA has been evaluated in three pivotal registration studies in naive patients receiving a variety of highly and moderately emetogenic chemotherapeutic agents [11-13]. The data in the clinical program provided evidence that NEPA plus dexamethasone provide superior CINV control over palonosetron plus dexamethasone in settings where a triple-drug combination is recommended. NEPA is now approved by the US FDA, EMA and other national agencies around the world, and is indicated for the prevention of acute and delayed nausea and vomiting associated with initial and repeat courses of cancer chemotherapy.

\section{Q Can you tell us more about anamorelin?}

Cancer anorexia-cachexia is a comorbidity of cancer. It is a multifactorial condition defined by an involuntary weight loss and sarcopenia, with or without loss of fat mass that cannot be fully reversed by conventional nutritional support and leads to progressive functional impairment. Ghrelin is an endogenous peptide secreted by the stomach that, upon binding to its receptor, stimulates multiple pathways in the positive regulation of body weight, lean body mass, appetite and metabolism.

Anamorelin is a novel, orally available, selective GRA that mimics the appetite-enhancing anabolic effects of ghrelin. It enables energy storage via gains in body weight (lean and fat mass), while also improving the symptoms and concerns related to cancer anorexia-cachexia.

We investigated the effects of anamorelin in two Phase III studies (ROMANA-1 and
ROMANA-2) in patients with advanced nonsmall-cell lung cancer experiencing anorexia and cachexia (defined as $>5 \%$ weight loss within prior 6 months or BMI $<20 \mathrm{~kg} / \mathrm{m}^{2}$ ). Anamorelin showed multiple benefits, not only increasing body weight (lean and fat mass) but also increasing appetite and reducing distress associated with anorexia and weight loss. Moreover, anamorelin administered daily over 12 and 24 weeks was well tolerated [14]. The drug is now in preregistration phase with EMA and other regulatory agencies, and new clinical studies are planned in different tumor types and in ancillary indications such as cancer fatigue.

Q You mentioned elsiglutide: can you tell us a little more about the rationale behind this program?

Elsiglutide, a 39 amino acid polypeptide, is a synthetic GLP-2 analog with potential intestinotrophic and antidiarrheal activities. In preclinical pharmacological studies, elsiglutide has shown to consistently stimulate growth of the small intestinal mucosa and decreases the incidence and severity of CID (data on file). Many cancer patients, who receive chemotherapy and in particular 5-fluorouracil-based chemotherapy but also certain targeted therapies or monoclonal antibodies, suffer from severe diarrhea induced by damages to their intestines through different mechanisms. Diarrhea is one of the most debilitating side effects associated with cancer treatment. CID is associated with weight loss, malnutrition, fatigue, loss of fluids and electrolytes imbalance that, in case of persistent and severe diarrhea, can result in life-threatening dehydration, renal insufficiency, and may contribute to cardiovascular morbidity. The occurrence of CID has a potential negative impact on the compliance of patients with their chemotherapy regimens and schedules, which can result in a delay in therapy, dosage reduction or discontinuation of therapy.

It is anticipated that the regenerative effects of elsiglutide may help ameliorate or prevent the destructive effects of chemotherapy on the small intestine and thereby reduce the incidence of diarrhea in cancer patients in chemotherapy. We recently completed a randomized, double-blind Phase IIb study in approximately 500 patients with colorectal cancer receiving 5-fluorouracilbased chemotherapy (folinic acid, 5-fluorouracil, oxaliplatin [FOLFOX] or folinic acid, 5-fluorouracil, irinotecan [FOLFIRI]) administered a single daily dose subcutaneously 
of elsiglutide 10, 20 or $40 \mathrm{mg}$ or placebo for 4 consecutive days. The primary end point was defined as the proportion of patients experiencing a maximum grade $\geq 2$ diarrhea during the first cycle of chemotherapy. In the trial, elsiglutide demonstrated a favorable tolerability profile; however, the primary end point was not met. Although the primary study outcome was not statistically significant, the pharmacological effect of elsiglutide was clearly shown and we are currently evaluating the best path forward for this program.

Q Helsinn's early-phase pipeline appears to be focusing on ghrelin receptor research for multiple indications: can you discuss this in more detail?

A few years ago Helsinn acquired the US company, Sapphire Therapeutics, which had a very attractive pipeline of drugs acting on the ghrelin receptor, including some interesting preclinical leads. In cooperation with a network of partners and service providers, we embarked on a lead optimization program aimed at identifying novel compounds acting on the ghrelin receptor as agonists, antagonists and inverse agonists. Our team was able to invent and develop HM01, the first brain-penetrant highly selective GRA, which is now being tested in ascending doses in a first-in-man study in healthy volunteers. Based on pharmacology studies, the most promising indication appears to be chemotherapy-induced neuropathic pain, a perfect fit with our supportive care focus. A second example coming out of these efforts is HM04, a highly selective ghrelin receptor antagonist with potential use in metabolic diseases and food disorders. The compound is completing Investigational New Drug Application-enabling studies and may proceed into human studies next year. Last, we have identified a few potential novel ghrelin inverse agonists which may be promoted to preclinical development in the next few months.

\section{Q Cancer supportive care is a} multidisciplinary approach: can you explain how Helsinn is helping to support \& achieve this?

A multidisciplinary assessment of cancer symptoms is important for comorbidity management, and can lead to treatment decision changes $5-40 \%$ of the time [15]. Ideally, the assessment team should include general practitioner, dietician, pharmacist, physiotherapist, specialist nurse and palliative/supportive care specialist. Unfortunately this does not occur in clinical practice. As a company primarily focused on supportive care, we try as much as possible to encourage educational initiatives to spread the concept of the importance of a multidisciplinary approach and its advantages for patients and caregivers. For example, we collaborate with scientific societies, such as the MASCC, to develop educational programs during the annual conference on this topic. Another key initiative in this direction is a long-term project in collaboration with the Bocconi University in Milan, aimed at supporting and developing mobile health technologies in cancer supportive care. Also the previously mentioned launch of the Helsinn Integrative Care business unit contributes in the holistic management of a wide range of cancer treatment side effects. Last but not least, support to patient organizations is another key aspect for increasing the awareness on the importance of a multidisciplinary approach in treating cancer therapy side effects.

\section{Q How is Helsinn collaborating with academia?}

Our relationships with the academic world are intense and occur at several levels. We have a number of research programs ongoing with university partners around the globe. For example, in our home country we are cooperating with the University of Lausanne in computational chemistry matters and with the University of Zürich in the testing of research molecules in highly specific animal models. Other examples include the sponsorship of a $\mathrm{PhD}$ student in Adelaide (Australia) working on chemotherapyinduced diarrhea pharmacology models, or the long-standing collaboration with a group at the Johns Hopkins University in Baltimore, MD, USA, on the mode of action of antiemetics.

With regard to clinical research, many of our large clinical studies involve academic centers. We recently started a strategic alliance on a broad, 5-year clinical program with The University of Texas MD Anderson Cancer Center in Houston, TX, USA. The program consists of 14 clinical trials in six different areas of cancer supportive and palliative care, namely fatigue, anorexia/cachexia, diarrhea, chronic nausea, pruritus and chemotherapy-induced neuropathic pain.

Last but not least, within the frame of our 'semivirtual' R\&D model as well as through the 
support of independent educational programs, we avail ourselves of the collaboration of a number of academicians in advisory roles.

\section{Q How does Helsinn work with patient} advocacy groups to support the oncology community?

As part of our patient-centered mission and support of the oncology community, we feel that partnering with patient advocacy groups is critical to achieving our corporate vision of improving patients' quality of life. We have developed close relationships with advocacy organizations in order to understand the oncology patients' unmet needs and work together to meet these needs. Patient advocacy groups protect the interests of cancer patients by helping them to receive appropriate and timely care, education, support and financial assistance, when needed. Helsinn is proud to provide funding and sponsorships to many of the oncology patient organizations who carry out these incredibly important goals.

\section{Q Where do you see Helsinn's role in cancer supportive care in 10 years' time?}

Supportive care in cancer is the prevention and management of the symptoms, and side effects of cancer and its treatment across the cancer continuum from diagnosis to the end of life. We at Helsinn believe that supportive care improves both quality of care and, consequently, quality of life of patients and caregivers, and this concept is fully aligned with our corporate mission to improve the everyday lives of cancer patients. As we are committed to building better todays for even more patients with cancer, we will continue our efforts in the supportive care arena, moving our pipeline forward very aggressively and seeking other promising opportunities. We will also continue to foster initiatives in supportive care that go beyond the development of promising drugs in areas of unmet medical need. These include, for example, the continuous improvement of our high-quality integrative care portfolio, the support of patient and professional organizations dedicated to supportive care like MASCC, funding of academic awards such as the Anna Braglia Endowed Young Investigator Award in Cancer Supportive Care at American Society of Clinical Oncology, mHealth initiatives, the previously mentioned alliance with MD Anderson among others.
Q Finally, do you believe a cure for cancer is a possibility?

The last decades have witnessed a formidable progress in cancer diagnosis and treatment, and in many instances cancer has evolved from being essentially a short-term death sentence to a chronic disease, and several cancer types are now curable. The most innovative approaches in immune-based and gene therapy (as well as all highly selective, mutation-specific drugs) bode very well for further improvements in cancer treatment outcomes, yet many settings remain extremely challenging and cures are not imaginable in the foreseeable future. A general misconception in the general public is that cancer is a unique disease that can potentially be addressed with a 'magic bullet' drug. Cancer biology and genetic research has demonstrated that this is totally wrong and future solutions will involve more and more personalized approaches, yet a cure in every patient and every tumor type remains, unfortunately, utopic.

\section{Q Do you have any closing remarks for our readers?}

Cancer remains a dreadful and often incurable disease and I encourage all readers to engage in the 'battle against cancer' in every possible way, always putting at the forefront the wellbeing and the dignity of the patients. Besides being active in industry or in the healthcare sector, which is of course not for everybody, a great deal can be achieved - for example, through the donation of time in the communities or through fundraising and sponsoring of organizations involved in cancer research and oncology patients care.

\footnotetext{
Disclaimer

The opinions expressed in this interview are those of the interviewee and do not necessarily reflect the views of Future Medicine Ltd.

Financial \& competing interests disclosure

$S$ Cantoreggi is an employee of Helsinn SA. Helsinn SA owns rights to products discussed within this interview. $S$ Cantoreggi has no other relevant affiliations or financial involvement with any organization or entity with a financial interest in or financial conflict with the subject matter or materials discussed in the manuscript apart from those disclosed.

No writing assistance was utilized in the production of this manuscript.
} 


\section{References}

1 Roila F, Tonato M, Ballatori E, Del Favero A. Prevention of cisplatin-induced emesis. Lancet 340(8825), 972 (1992).

2 Joss RA, Bacchi M, Buser K et al. Ondansetron plus dexamethasone is superior to ondansetron alone in the prevention of emesis in chemotherapy-naive and previously treated patients. Ann. Oncol. 5(3), 253-258 (1994).

3 Rojas C, Stathis M, Thomas AG et al. Palonosetron exhibits unique molecular interactions with the $5-\mathrm{HT}_{3}$ receptor. Anesth. Analg. 107(2), 469-478 (2008).

4 Eisenberg P, Figueroa-Vadillo J, Zamora R et al. Improved prevention of moderately emetogenic chemotherapy-induced nausea and vomiting with palonosetron, a pharmacologically novel $5-\mathrm{HT}_{3}$ receptor antagonist: results of a Phase III, single-dose trial versus dolasetron. Cancer 98(11), 2473-2482 (2003).

5 Gralla R, Lichintser M, Van Der Vegt S et al. Palonosetron improves prevention of chemotherapy nausea and vomiting following moderately emetogenic chemotherapy: results of a double blind randomized Phase III trial comparing single doses of palonosetron with ondansetron. Ann. Oncol. 14(10), 1570-1577 (2003).

6 Saito M, Aogi K, Sekine I et al. Palonosetron plus dexamethasone for prevention of nausea and vomiting during chemotherapy: a double blind, double dummy, randomised, comparative Phase III trial. Lancet Oncol. 10(2), 115-124 (2009).

7 Hesketh PJ, Bohlke K, Lyman GH et al. Antiemetics: American Society of Clinical Oncology focused guideline update. J. Clin. Oncol. 34(4), 381-386 (2016).

8 MASCC antiemetic guidelines. www.mascc.org

$9 \mathrm{NCCN}$ antiemesis guidelines. www.nccn.org

10 Aapro M, Molassiotis A, Dicato M et al. The effect of guidelines-consistent anti-emetic therapy on chemotherapy-induced nausea and vomiting (CINV): the Pan European Emesis Registry (PEER). Ann. Oncol. 23(8), 1986-1992 (2012).

11 Hesketh PJ, Rossi G, Rizzi G et al. Efficacy and safety of NEPA, an oral combination of netupitant and palonosetron, for prevention of chemotherapy-induced nausea and vomiting following highly emetogenic chemotherapy: a randomized dose-ranging pivotal study. Ann. Oncol. 25(7), 1340-1346 (2014).

12 Aapro M, Rugo H, Rossi G et al. A randomized Phase III study evaluating the efficacy and safety of NEPA, a fixed dose combination of netupitant and palonosetron, for prevention of chemotherapy induced nausea and vomiting following moderately emetogenic chemotherapy. Ann. Oncol. 25(7), 1328-1333 (2014).

13 Gralla R, Bosnjak SM, Hontsa A et al. A Phase III study evaluating the safety and efficacy of NEPA, a fixed dose combination of netupitant and palonosetron, for prevention of chemotherapy-induced nausea and vomiting over repeated cycles of chemotherapy. Ann. Oncol. 25(7), 1333-1339 (2014).

14 Temel JS, Abernethy AP, Currow DC et al. Anamorelin in patients with non-small-cell lung cancer and cachexia (ROMANA 1 and ROMANA 2): results from two randomised, double-blind, Phase III trials. Lancet Oncol. 17(4), 519-531 (2016).

15 Wright FC, De Vito C, Langer B, Hunter A. Multidisciplinary cancer conferences: a systematic review and development of practice standards. Eur. J. Cancer 43(6), 1002-1010 (2007). 